\title{
Theoretical Aspects of Numerical Identification ${ }^{1}$
}

\author{
L. R. HILL \\ National Collection of Type Cultures, Central Public Health Laboratory, London NW9 SHT, England
}

\begin{abstract}
All identification processes are strictly probabilistic. Identification allocates initially unidentified operational taxonomic units (U-OTUs) to taxa contained in previously established classifications. Identification is therefore subject to a number of a priori conditions, such as the probabilistic nature of classifications, the manner in which classifications are made, the adequacy of classifications, and the adequacy of the set of characters describing U-OTUs. The advantages and disadvantages of continuous reclassification, incorporating U-OTUs, are discussed. Numerical identification is concerned with extracting from a classification a minimum, or near minimum, amount of information necessary to effect separation of all the taxa defined by the classification. Several strategies are available for achieving this-test reduction, OTU reduction, a combination of both of these, and key generation.
\end{abstract}

It might be useful to begin at the beginning and reiterate the essential difference between classification and identification.

Classification is a process whereby one seeks to give a taxonomic, i.e., grouped, structure to sets of organisms which a priori lack such structure and are unordered or unsorted. Usually, and fundamental to those classifications based on concepts of overall similarity, namely numerical classifications, the objective is to divide and group the set of organisms so that within-group variance in properties of the organisms will be less than between-group variance.

On the other hand, identification is a process whereby an organism of unknown taxonomic affinity can be allocated, or said "to belong" to a preexisting known taxonomic group, the allocation being determined by the study of its properties.

Conceptually, classification is an academic exercise, often with a practical end in view, whereas identification is a wholly practical necessity. Since identification can only operate within the context of a preexisting classification, it is subject to a number of a priori conditions or constraints, among which perhaps the most important are (i) classifications are probabilistic, (ii) the manner by which classifiqations are made, (iii) the adequacy of the set of properties known about the unidentified operational taxonomic units (U-OTU), and (iv) the adequacy of the classification.

Because of these and doubtless other a priori

\footnotetext{
'Text of a paper read at the 1st International Congress for Bacteriology, Taxonomy Session, Jerusalem, 3 September 1973.
}

conditions affecting identification, it follows that any identification method, numerical or otherwise, is strictly probabilistic. There can never be an absolute finality to an identification. The problem is simply to discriminate between taxa in a classification to find that taxon to which the U-OTU is most likely to belong.

The first constraint, that classifications are themselves probabilistic, is self-evident in the case of numerical taxonomies, and it has been previously emphasized (18) that the result of numerical classification should be regarded as no more than a hypothesis, like any other scientific hypothesis, to be subjected to further testing and modification or even outright rejection, as new evidence becomes availab̆le. One may think, on the other hand, that non-numerical taxonomies are non-probabilistic, but this is not so-even the most conventionally devised classification can only be the result of certain data available. Unused available data, or new data, can and most likely will have an effect on the classification. Thus, a numerical or non-numerical classification context within which identification operates is not and cannot be a firm, fixed background. At no one time, when an identification is being attempted, can the relevant classification be said to be absolute or final.

In regards to the second constraint, i.e., how classifications are made, it is obvious that numerical classifications provide the best base for numerical identification, as the classification itself will contain numerical estimates of variance and frequency of positive or negative responses to particular tests in particular taxa. The choice of a numerical identification 
method can be influenced by the numerical taxonomy method already used. However, devising a numerical identification method need not be limited only to groups classified by numerical taxonomy techniques. Provided that quantitative or semi-quantitative data regarding frequency of responses are available, even for groups defined in a context of conventional classification, a numerical identification method then becomes a practicable proposition. Few of the taxa contained in the range currently identifiable at the Colindale laboratory by the method of Lapage (8) have been studied by numerical taxonomy. However, they are taxa which are relatively well-documented in the literature and, therefore, that particular numerical identification method was possible.

The amount known about the U-OTU, the third constraint, is generally less than the amount known about the OTUs used to devise the classification. The identification is therefore carried out partly in ignorance. This may be no great handicap in a step-wise identification method, for although I subscribe to the view that all characters are equally important to estimate paired similarities in a numerical taxonomy method, I do not agree that they are all equally important in determining the resultant classification. Numerical taxonomies, i.e., actual arrangements of OTUs in groups, are determined by highly correlated characters; poorly correlated or uncorrelated characters have little or no effect on the classification. Thus, in a step-wise identification, ideally one would determine for the U-OTU those characters that were important for the classification. This ideal cannot always be attained, since quite exotic characters or those difficult to determine may have been justifiably used in the classification and may also be important a posteriori, but it may not be justifiable to determine them for each U-OTU. More generally, however, one is presented with some results for the U-OTU, and one must do his best with that.

Regarding the fourth constraint, the adequacy of the classification, there is always the possibility that the partially described U-OTU may belong to a taxon not represented in the classification at all. In making a numerical identification method, provision should be made for this event, but this is not easy. The method can be prefaced, as it were, by a listing of all those characters common to all the taxa catered for, but that may not always exclude a stranger. Another provision, incorporated in the method described by Lapage (8), is to attach to the identification a probability measure. Thus, the identification method will indicate the most likely taxon for the U-OTU among those taxa available, but with a low probability score, indicating that the U-OTU is really a stranger.

Turning to methods of numerical identification, the first most obvious way is simply reclassification of a system with the U-OTUs now included. This is not of ten done strictly as an identification method, but in practice most numerical taxonomies contain both OTUs with fairly certain identities, and others, generally "field isolates," which are U-OTUs. Such numerical taxonomies can be regarded as numerical identifications as far as the U-OTUs are concerned. The advantages of reclassification are several: ready availability of numerical taxonomy programs, continuous taxonomic revision, and satisfactory identification as a new taxon of those U-OTUs not belonging to any previous taxon contained in the classification. Generally, however, the problem is to identify just one or a few U-OTUs and so the advantages become outweighed by certain disadvantages: classification is neither quick (not even with computers) nor economical; acceptable identification can usually be made from the fewer characters known for the U-OTU than are necessary for reclassification; U-OTUs usually belong to an existing group which may be sufficiently well defined not to require continual revision.

Apart from reclassification, numerical identification is more usually concerned with extracting from a classification a minimum, or near minimum, amount of information necessary to effect separation of all the taxa defined by the classification. This can be achieved by reducing the number of tests, or the number of OTUs in the classification, or both, or by generating keys.

Before discussing these approaches, I would like to mention one mental identification procedure not amenable to numerical identification and where computers cannot help, at least at present. This is "immediate recognition" or, perhaps, better known in microbiology, "spot identification." Morse (12) suggests that in such cases the mind may not be using any repetitive elimination procedures, and so spot identification is beyond present computer capability. I mention this because it exemplifies another way of looking at numerical identification, namely as procedures of progressive elimination of possible identifications.

\section{TEST REDUCTION}

Methods of test reduction use various measures of the content of information of tests in 
order to select the most informative ones. "Information content" was originally defined by Rescigno and Maccacaro (16) in their repartition value $(H)$. Basically, the idea behind this measure and those mentioned below (see also Appendix) is that a test which subdivides the OTUs into equal subsets has a higher information content than one dividing the OTUs into unequal subsets. Möller (11) has devised a correction factor for $\mathrm{H}$ to allow for missing data, and extended $\mathrm{H}$ itself to consider tests in pairs, triplicates, and the like.

A simpler measure, but equally effective in practice, is Gyllenberg's (2) separation figure (S). Gower and Barnett's (1) measure $\left(\mathrm{OT}^{2}\right)$ is virually the same, but also allows for missing data. Rypka et al. (17) extended Gyllenberg's separation figure to consider tests in pairs, triplicates, and the like.

Another measure is the identification index (I) of Niemelä et al. (14), whereas Pankhurst's (15) measure $(F)$ is probably the most advanced one and was developed to consider tests that may be binary or multiple, weighted or unweighted, and considers missing data.

By any of these measures, tests (or pairs of tests, triplicates, and the like) can be ranked in order of decreasing importance. An identification matrix of OTUs versus tests can be built up starting with the highest ranking test and descending the reordered sequence until all OTUs are separated. Alternatively, these calculations can be made to generate keys (vide infra). Matrix reduction was also achieved by Niemalä et al. (14) by continuous deletion from the original classificatory OTU versus tests matrix. Matrix entries are converted to +1 and -1 values and added algebraically for each test, and that test with the highest sum is deleted. The matrix is then examined to check if all OTUs are still different from each other and, if so, the next highest test is removed, and so on, until removal of a test results in some OTUs becoming identical. The last test is therefore retained and so finally the OTUs are described by a minimum set of tests.

\section{IDENTIFICATION KEYS}

Much has been written about identification keys $(10,12,13,15)$, and some common themes can be discerned. The most important of these themes appear to be the following.

(i) There is necessity for a device to establish a priori whether the U-OTU does belong to a taxon included in the key. Keys can be perfaced with a listing of characters common to all taxa.

(ii) A key should give, on the average, the shortest path to identification. In the case of bacteriological identification, this is not an important consideration. Bacteriological keys are not used in the same way as, for example, botanical keys. With the latter, U-OTU properties are determined one by one as the key progresses. Since most bacteriological properties require time for their determination, a set of properties is determined before turning to a key.

(iii) Variable characters should preferably not be used. If they must be used, then either probabilities can be utilized, or taxa can be keyed-out several times instead of once.

(iv) Keys should be supplemented at their termini by that information not used in the key itself.

(v) Tests used in a key should be those with the highest information content. (Since this is equivalent to subdividing into equal, or near equal, subsets at each stage, items ii and $v$ are achieved simultaneously.)

(vi) Before devising a key, consideration should be given to the desirability of first modifying the raw data from which the key is to be made. For numerical taxonomy, all characters have equal a priori weight: they then acquire different weights in determining the classification (5). It is facile, but erroneous, to assume that the importance thus acquired by different tests is equivalent to importance for use in an identification key. For identification, there are other, new conditions to be considered, ranging from such practicalities as ease of determining particular tests (4) to a priori likelihood of a particular taxon to occur (11).

Bearing these themes in mind, keys are constructed by using the various measures of information content mentioned above to choose that test (pair of tests, triplicates, and the like) to effect a first subdivision of the OTUs into subsets. The calculations are repeated for each remaining test with each subset separately, and so on, to give the branching sequence of keys.

\section{MEDIAN ORGANISMS}

Finally, a considerable saving in the amount of information to be stored as reference data can be achieved if taxa are replaced by single rows of information which will be representative of the taxa. Such thinking has a long and 
respected tradition in biology, for essentially the practice of retaining type cultures for posterity is the same, although there is no requirement that a nomenclatural type culture be typical.

A number of authors have proposed solutions to this end. Silvestri et al. (19) labeled those strains around which taxa were formed as "centrotypes," or simply those OTUs which had the highest average similarly with all members of their taxa. This approach has the advantage of giving an actual organism as the reference data for a taxon. Liston et al. (9) developed the concept of a "hypothetical median organism" (see also reference 21). Gyllenberg (3) suggested that, for factor analysis methods of numerical taxonomy (7), once taxa are defined in their multidimensional space, respective centers of gravity of taxa can be computed. To precisely compare a U-OTU, one would have to determine all the defining characters, as used in the taxonomy. It is probable, however, that one could use only those characters heavily weighted in the particular factors used to define the space in which the taxa are delimited, although it appears that experiments in this direction have not been attempted.

The disadvantages of comparing a U-OTU with a set of centrotypes, median organisms, or centers of gravity is that these respective reference OTUs cannot at the same time represent the range of variability of their respective taxa.

\section{CONCLUSION}

The probabilistic nature of identification has been emphasized; increasing awareness of this aspect is probably due to the development of numerical taxonomy. The broad approaches currently available for numerical identification, arising out of numerical taxonomy, have been outlined. At this point in time there is little to say about the comparative merits and defects of these approaches because, although a considerable body of numerical taxonomy literature has appeared, the same is not true of numerical identification. Some years of further experimentation and use must take place to provide the necessary evidence against which these approaches can be assessed.

Some comment can be made, however, about the type of information and kinds of developments which will be necessary to allow progress in the field of numerical identification.
Future development of numerical identification should not be limited in any way by computing and programming constraints; the problems here are no greater than for numerical taxonomy. Rather, the future development will be limited at the biological level. Quantitative estimates of frequencies of responses to tests for particular taxa are needed. These would immediately permit the construction of other input matrices and creation of numerical identification schemes, such as that for gramnegative rods described by Lapage (8), for other groups.

Secondly, more work appears necessary at the mathematical level. There is no lack of measures for estimating the relative importance of tests, but little has been done on usefully describing the range and variability of taxa. Tsukamura (20) gives a calculation of the standard deviation for numerically defined taxa, but more than this single statistic is needed. The variability must be expressed in terms of tests.

Finally, those engaged in numerical taxonomy studies should be encouraged to add numerical identification sections to their works.

\section{APPENDIX}

This appendix gives the formulas of those measures of the relative importance of tests, for identification purposes, that were mentioned above. As different authors used different symbols, the formulas have been rewritten with a set of standard symbols given in Table 1. The rewritten formulas, and some general properties of the measures, are given in Table 2. Numerical values of these measures for the hypothetical matrix of Table 1 are given in Table 3. In some

TABLE 1. Symbols used to rewrite the formulas of the proposed measures

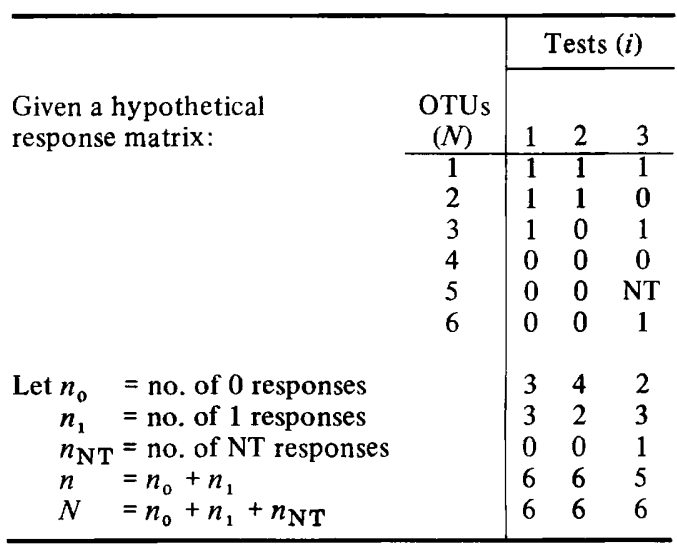


TABLE 2. Formulas of the proposed measures and some of their general properties

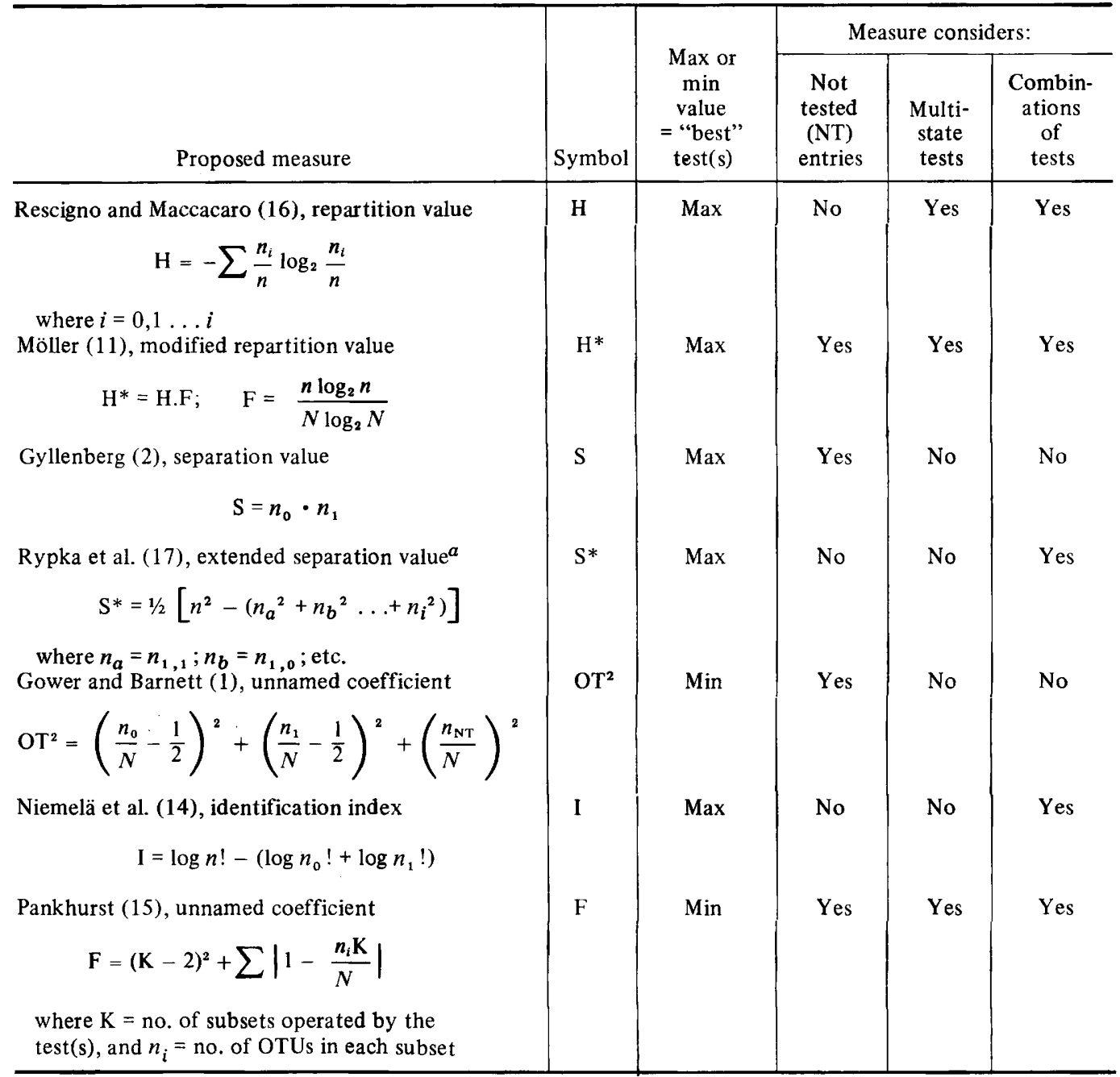

TABLE 3. Calculated values of the proposed measures for the hypothetical matrix

\begin{tabular}{l|l|l|l|l|l|l}
\hline \multirow{2}{*}{$\begin{array}{c}\text { Proposed } \\
\text { measure }\end{array}$} & \multicolumn{3}{|c|}{ Single tests } & \multicolumn{3}{c}{ Combinations of tests } \\
\cline { 2 - 7 } & 1 & 2 & 3 & 1,2 & 1,3 & 2,3 \\
\hline $\mathrm{H}$ & 1 & 0.92 & $0.97^{a}$ & 1.46 & $1.92^{a}$ & $1.92^{a}$ \\
$\mathrm{H}^{*}$ & 1 & 0.92 & 0.73 & 1.46 & 1.44 & 1.44 \\
since F & 1 & 1 & 0.75 & 1.00 & 0.75 & 0.75 \\
$\mathrm{~S}$ & 9 & 8 & 6 & & 9 & $9^{a}$ \\
$\mathrm{~S}^{*}$ & 0 & 0.056 & 0.056 & 11 & $9^{a}$ & $1.778^{a}$ \\
$\mathrm{OT}^{2}$ & 1.301 & 1.176 & $1.000^{a}$ & 1.778 & $1.778^{a}$ & 6.00 \\
$\mathrm{I}$ & 0 & 0.67 & 0.33 & 2.00 & 5.33 & 4 \\
$\mathrm{~F}$ & 2 & 2 & 2 & 3 & $4,1,3,2$ \\
since K & 3,3 & 4,2 & 3,4 & $2,1,3$ & $2,1,2,2$ & $1,1,3$ \\
and $n_{i}$ & 3,3 &
\end{tabular}

\footnotetext{
${ }^{a}$ Theoretical value only, since corresponding measure should not be used when NT entries are present.
} 
instances, numerical values have been calculated for a particular measure when, in fact, it would be inappropriate to use that measure because of NT (not tested, or missing data) entries in the matrix. They are included solely for illustrative purposes.

\section{ACKNOWLEDGMENT}

I wish to thank W. R. Willcox for helpful discussion and comment.

\section{REPRINT REQUESTS}

Address requests for reprints to: Mr. L. R. Hill, National Collection of Type Cultures, Central Public Health Laboratory, Colindale Avenue, London, NW9 5HT, England.

\section{LITERATURE CITED}

1. Gower, J. C., and J. A. Barnett. 1971. Selecting tests in diagnostic keys with unknown responses. Nature (London) 232:491-493.

2. Gyllenberg, H. G. 1963. A general method for deriving determination schemes for random collections of microbial isolates. Ann. Acad. Sci. Fenn. Ser. A4 69:1-23.

3. Gyllenberg, H. G. 1965. A model for computer identification of microorganisms. J. Gen. Microbiol. 39:401-405.

4. Hall, A. V. 1970. A computer-based system for forming identification keys. Taxon 19:12-18.

5. Hill, L. R. 1972. Prospectives for Mycoplasma classification using multivariate analysis methods. Med. Microbiol. Immunol. 157:101-112.

6. Hill, L. R., and L. G. Silvestri. 1962. Quantitative methods in the systematics of actinomycetales. III. The taxonomic significance of physiologicalbiochemical characters and the construction of a diagnostic key. Gen. Microbiol. 10:1-28.

7. Hill, L. R., L. G. Silvestri, P. Ihm, G. Farchi, and P. Lanciani. 1965. Automatic classification of staphylococci by principal-component analysis and a gradient method. J. Bacteriol. 89:13931401 .

8. Lapage, S. P. 1974. Practical aspects of probabilistic identification of bacteria. Int. J. Syst. Bacteriol. 24:500-507.
9. Liston, J., W. Wiebe, and R. R. Colwell. 1963. Quantitative approach to the study of bacterial species. J. Bacteriol. 85:1061-1070.

10. Metcalf, Z. P. 1954. The construction of keys. Syst. Zool. 3:38-45.

11. Möller, F. 1962. Quantitative methods in the systematics of actinomycetales. IV. The theory and application of a probabilistic identification key. Gen. Microbiol. 10:29-47.

12. Morse, L. E. 1971. Specimen identification and key construction with time-sharing computers. Taxon 20:269-282.

13. Niemelä, S. I., and H. G. Gyllenberg. 1968. Application of numerical methods to the identification of micro-organisms. Spisy Prirodoved. Fak. Univ. Brne 43: Series K, p. 279-289.

14. Niemelä. S. I., J. W. Hopkins, and C. Quadling. 1968. Selecting an economical binary test battery for a set of microbial cultures. Can. J. Microbiol. 14:271-279.

15. Pankhurst, R. J. 1970. A computer program for generating diagnostic keys. Computer J. 13:145151.

16. Rescigno, A., and G. A. Maccacaro. 1961. The information content of biological classifications, p. 437-446. In C. Cherry (ed.), Information theory-a symposium held at the Royal Institution, London, 1960. Butterworth, London

17. Rypka, E. W., W. E. Clapper, I. G. Bowen, and R. Babb. 1967. A model for the identification of bacteria. J. Gen. Microbiol. 46:407-424.

18. Silvestri, L. G., and L. R. Hill. 1964. Some problems of the taxometric approach, p. 87-103. In V. H. Heywood and J. McNeill (ed.), Phenetic and phylogenetic classification, The Systematics Association, Publication No. 6, London.

19. Silvestri, L. G., M. Turri, L. R. Hill, and E. Gilardi. 1962. A quantitative approach to the systematics of actinomycetes based on overall similarity, p. 333-360. In G. C. Ainsworth and P. H. A. Sneath (ed.), Microbial classification, 12th Symposium of the Society for General Microbiology. Cambridge University Press, Cambridge.

20. Tsukamura, M. 1967. A statistical approach to the definition of bacterial species. Jap. J. Microbiol. 11:213-220.

21. Tsukamura, M., and S. Mizuno. 1968. "Hypothetical mean organisms" of mycobacteria. A study of classification of mycobacteria. Jap. J. Microbiol. 12:371-384. 